\title{
Inverse melting and inverse freezing in a three-state spin-glass model with finite connectivity
}

\author{
R. Erichsen, Jr.* and W. K. Theumann ${ }^{\dagger}$ \\ Instituto de Física, Universidade Federal do Rio Grande do Sul, Caixa Postal 15051, 91501-970 Porto Alegre, RS, Brazil \\ S. G. Magalhaes $s^{\ddagger}$ \\ Instituto de Fisica, Universidade Federal Fluminense, 24210-346 Niterói, RJ, Brazil
}

(Received 17 September 2012; published 24 January 2013)

\begin{abstract}
The phase diagrams of the three-state Ghatak-Sherrington spin-glass (or random Blume-Capel) model are obtained in mean-field theory with replica symmetry in order to study the effects of a ferromagnetic bias and finite random connectivity in which each spin is connected to a finite number of other spins. It is shown that inverse melting from a ferromagnetic to a low-temperature paramagnetic phase may appear for small but finite disorder and that inverse freezing appears for large disorder. There can also be a continuous inverse ferromagnetic to spin-glass transition.
\end{abstract}

DOI: 10.1103/PhysRevE.87.012139

PACS number(s): 64.60.De, 87.19.1j, 87.19.1g

Versions [1-6] of the Blume-Emery-Griffiths (BEG) model [7] with random interactions have been studied lately as spin models that yield thermodynamic phase diagrams exhibiting unusual reversible inverse transitions (IT), as inverse melting and inverse freezing, that have been found experimentally in a variety of quite different systems. Examples in which they occur are $\mathrm{He}^{3}$ and $\mathrm{He}^{4}$ isotopes at very low temperature and high pressure, the polymer P4MP1, solutions, colloidal systems, ferroelectricity in Rochelle salt, ferromagnetism of gold nanoparticles, high-temperature superconductors, and quantum-spin systems [8] (see Refs. [4,6] for a recent summary of references).

Inverse melting appears as the reversible first-order transition between a liquid or completely disordered paramagnetic phase at low temperature and a crystalline or ordered phase at higher temperature, whereas inverse freezing is the reversible first-order transition from a paramagnetic phase to a hightemperature amorphous or glassy phase [4,6]. These transitions usually appear with a reentrance in the phase boundary of continuous transitions at high temperature between an ordered and a fully disordered phase. The characteristic feature of inverse transitions is that the ordered high-temperature phase is favored by the entropy while the low-temperature disordered phase is favored by the minimum of the energy. This is best illustrated by the change of the folded into the unfolded configurations of methyl cellulose polymer chains in water. The bundles of methyl groups that are folded in a compact weakly interacting configuration at low $T$ unfold with increasing $T$ making more microscopic configurations available with an increase in volume and entropy $[4,9]$.

The three-state spin-glass (SG) model with a crystal-field term of Ghatak and Sherrington (GS) [1] is a Blume-Capel (BC) model $[10,11]$ with random bonds that exhibits a continuous transition between a spin-glass and a paramagnetic (P) phase at high temperature and a reentrant phase boundary at low temperature. Inverse freezing appears on the latter as a

\footnotetext{
*rubem@if.ufrgs.br

†theumann@if.ufrgs.br

${ }^{\ddagger}$ sgmagal@gmail.com
}

genuine thermodynamic first-order transition below a tricritical point in mean-field theory with infinite-range interactions and full replica symmetry breaking (FRSB) [3]. Similar results were obtained in mean-field theory with one-step replica symmetry breaking for the $\mathrm{BC}$ model with spin degeneracy [4]. Inverse freezing also appears in numerical simulations for a three-dimensional GS model with nearest-neighbor interactions [6] and numerical work on the two-dimensional randombond Ising model exhibits an inverse melting transition [12]. It would be interesting to have independent analytical results for either of these random systems with finite-range interactions exhibiting both IT.

Theoretical works on inverse freezing deal usually with a symmetric distribution of random bonds in fully connected systems. The purpose of the present work is to study, by means of an analytical procedure combined with a numerical evolution of a population dynamics [17], the dependence on disorder of the phase diagrams for the GS model with finite connectivity as a way to account for effective finite-range interactions [13-16], and in order to achieve this we use an asymmetric distribution of random bonds.

The three-state spin-glass model of Ghatak and Sherrington with finite connectivity is described by the Hamiltonian

$$
H=-\frac{1}{c} \sum_{i<j} c_{i j} J_{i j} \sigma_{i} \sigma_{j}+D \sum_{i} \sigma_{i}^{2},
$$

with the spins $\sigma_{i} \in\{-1,0,1\}, i=1 \ldots N$ and the random symmetric $\left(c_{j i}=c_{i j}\right)$ connectivity variable $c_{i j}$. The latter takes the values $c_{i j}=1$ if there is a connection between a pair of spins at sites $(i j)$ and $c_{i j}=0$ if there is none, independently of other pairs of spins, according to a Poisson distribution for small $c / N$

$$
p\left(c_{i j}\right)=\frac{c}{N} \delta_{c_{i j}, 1}+\left(1-\frac{c}{N}\right) \delta_{c_{i j}, 0} .
$$

The network connectivity $c$, that is the average number of connections per site, is assumed to remain finite in the thermodynamic limit $N \rightarrow \infty$, such that $c / N \rightarrow 0$, and the statistical mechanics for the model is derived in that limit. The set of infinite-range interactions $\left\{J_{i j}\right\}$ is assumed to consist of independent, identically distributed random variables 
drawn from the binary distribution $p\left(J_{i j}\right)=p \delta\left(J_{i j}-J\right)+$ $(1-p) \delta\left(J_{i j}+J\right)$ for every pair of sites $(i, j)$, where $J>0$. Thus, each coupling has a probability $p$ to be ferromagnetic and $(1-p)$ to be antiferromagnetic, where $(1-p)$ plays the role of a quenched disorder. For simplicity, we take $J=1$. Finally, the quadratic form in the spins favors the population of the zero state, if $D>0$, or the states \pm 1 , if $D<0$.

Assuming thermal equilibrium at an inverse temperature $\beta=1 / T$, the disorder-averaged free energy per spin is calculated in the replica procedure as [18]

$$
f(\beta)=-\lim _{N \rightarrow \infty} \frac{1}{\beta N} \lim _{n \rightarrow 0} \frac{1}{n} \log \left\langle Z^{n}\right\rangle,
$$

where $n$ is the number of replicas, $Z=\sum_{\sigma_{1} \ldots \sigma_{N}} \mathrm{e}^{-\beta H}$ is the partition function, and the brackets stand for the disorder average over the sets of both $c_{i j}$ and $J_{i j}$, in that order. In the small $c / N$ limit, the disorder-average replicated partition function becomes, to leading order in $N$,

$$
\begin{aligned}
\left\langle Z^{n}\right\rangle= & \sum_{\boldsymbol{\sigma}^{1} \ldots \boldsymbol{\sigma}^{n}} \exp \left[-\beta D \sum_{i, \alpha}\left(\sigma_{i}^{\alpha}\right)^{2}\right. \\
& \left.+\frac{c}{2 N} \sum_{i \neq j}\left\langle\mathrm{e}^{\frac{1}{c} \beta J \sum_{\alpha} \sigma_{i}^{\alpha} \sigma_{j}^{\alpha}}-1\right\rangle_{J}\right],
\end{aligned}
$$

where $\alpha=1, \ldots, n$ denotes the replica index. Here, $\langle g(J)\rangle_{J}$ is the average of an interaction-dependent quantity $g\left(J_{i j}\right)$ over the distribution $p\left(J_{i j}\right)$.

Since the connectivity $c$ is finite, one cannot expand the inner exponential and follow the standard infinite-connectivity procedure. Instead, we refer to Ref. [16] for a detailed calculation of the finite-connectivity replica symmetric (RS) solution for the three-state spin-glass problem. This requires the introduction of an order function $P(\sigma)=(1 / N) \sum_{i} \delta \boldsymbol{\sigma} \sigma_{i}$, which represents the fraction of sites with the replica configuration given by the $n$-component vector $\sigma$, where $\delta_{\sigma} \sigma_{i}=1$ if $\sigma=\sigma_{i}$ and zero otherwise. The order function follows a saddle-point equation and to solve it we restrict the present work to a RS ansatz and assume that [16]

$$
P(\boldsymbol{\sigma})=\int d h d b W(h, b) \frac{\mathrm{e}^{\beta h \sum_{\alpha} \sigma_{\alpha}-\beta b \sum_{\alpha} \sigma_{\alpha}^{2}}}{\left[2 \mathrm{e}^{-\beta b} \cosh (\beta h)+1\right]^{n}},
$$

for any real $n$, where $h$ and $b$ are the two components of a local field distributed according to the density $W(h, b)$ that has to be determined self-consistently. For each temperature $T=1 / \beta$, crystalline field $D$, and connectivity $c$, we proceed numerically by means of an iterative population dynamics procedure for a large number of vector fields. As an initial guess, a uniform distribution of fields is chosen, which are then updated as follows [17]. First, a number $k$ is chosen from a Poisson distribution of mean $c$. Then, cells $\left(h_{l}, b_{l}\right)$ with $l$ running from 1 to $k$ are selected randomly from the population, while couplings $J_{l}$ are selected from the binary distribution $p\left(J_{l}\right)$ given above with $J_{l}$ replacing $J_{i j}$. Next, one selects randomly a new cell $(h, b)$ from the population and sets $h=\frac{1}{\beta} \sum_{l=1}^{k} \phi\left(h_{l}, b_{l}, J_{l}\right)$ and $b=\theta-\frac{1}{\beta} \sum_{l=1}^{k} \psi\left(h_{l}, b_{l}, J_{l}\right)$, where

$$
\phi(h, b, J)=\frac{1}{2} \log \frac{2 a_{1}+\mathrm{e}^{\beta b}}{2 a_{-1}+\mathrm{e}^{\beta b}}
$$
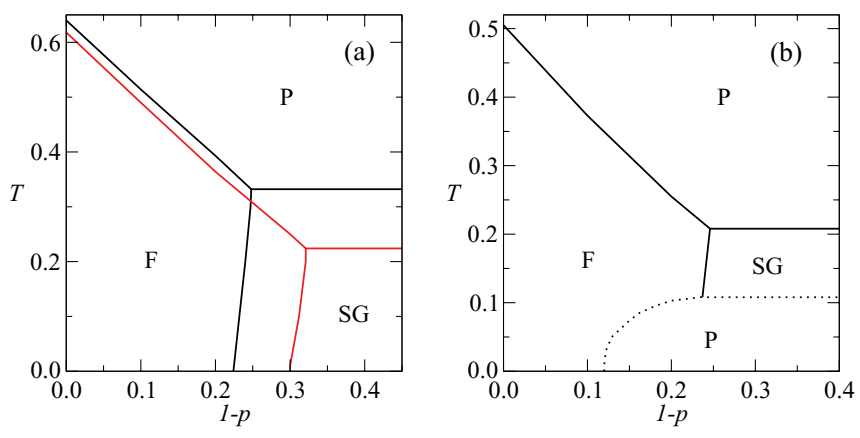

FIG. 1. (Color online) Phase diagram $T$ vs. disorder $1-p$. (a) $D=0.2, c=4$ (black lines), and $c=8$ (red lines). (b) $D=0.48$, $c=4$. Solid (dotted) lines correspond to continuous (discontinuous) transitions.

and

$$
\psi(h, b, J)=\frac{1}{2} \log \frac{\left(2 a_{1}+\mathrm{e}^{\beta b}\right)\left(2 a_{-1}+\mathrm{e}^{\beta b}\right)}{\left[2 a_{0}+\mathrm{e}^{\beta b}\right]^{2}}
$$

follow from the saddle-point equation for the order function in which $a_{\sigma}=\cosh (\beta h+\sigma \beta J / c)$ is the part that contains the interaction and, for simplicity of notation, we left out the cell label $l$. The procedure is continued until it converges to a limiting $W(h, b)$.

Knowledge of $W(h, b)$ allows us to determine directly the magnetization $m=\int d h d b W(h, b)\langle\sigma\rangle$, the spin-glass order parameter $q=\int d h d b W(h, b)\langle\sigma\rangle^{2}$, and the activity, $r=\int d h d b W(h, b)\left\langle\sigma^{2}\right\rangle$. The free energy and the entropy per site, needed for the results presented below, were also obtained in the procedure [16]. As usual in the three-state model, $m \neq 0$, $q>0$, and $r>0$ indicates magnetic order; $m=0, q>0$, and $r>0$ characterizes spin-glass ordering and $m=0=q$ and $r \geqslant 0$ describes paramagnetic order. The activity parameter $r$ is zero when all spins are in the $\sigma_{i}=0$ local state. Otherwise, it is a variable with finite nonzero value.

We present next the results for the phase diagrams. In Fig. 1 we show the domains of a ferromagnetic phase $(\mathrm{F})$, a spin-glass phase (SG), and a paramagnetic phase $(\mathrm{P})$. The latter may appear even at low $T$ if the crystal field favoring inactive spin states is large enough. If $D$ is small, most spins are expected to be active, that is in the states \pm 1 , and the nature of the phase should depend strongly on the amount of disorder $1-p$. Small disorder favors $\mathrm{F}$ states and large disorder favors SG states, which is what one would expect for a given connectivity as shown in Fig. 1(a) for $c \geqslant 4$. That $c$ is the smallest value of the connectivity for which the continuous $\mathrm{SG}=\mathrm{P}$ phase boundary has a reentrance at all. As $D$ is increased, favoring inactive spins, one would still expect stable ferromagnetic states at low but finite disorder that should disappear with increasing disorder. What is new is that, instead of a SG phase, a lowactivity (small-r) $\mathrm{P}$ phase appears at low $T$ for not too small disorder, as can be seen in Fig. 1(b). These effects of the crystal field should be reduced at higher $T$, leaving a region of $\mathrm{F}$ states for small disorder followed by a region of SG states for higher disorder.

Thus, for low but increasing $T$ with appropriate values of $D$ and a small but finite disorder $(1-p)$, one may expect an inverse melting transition from the low- $T$ paramagnetic 

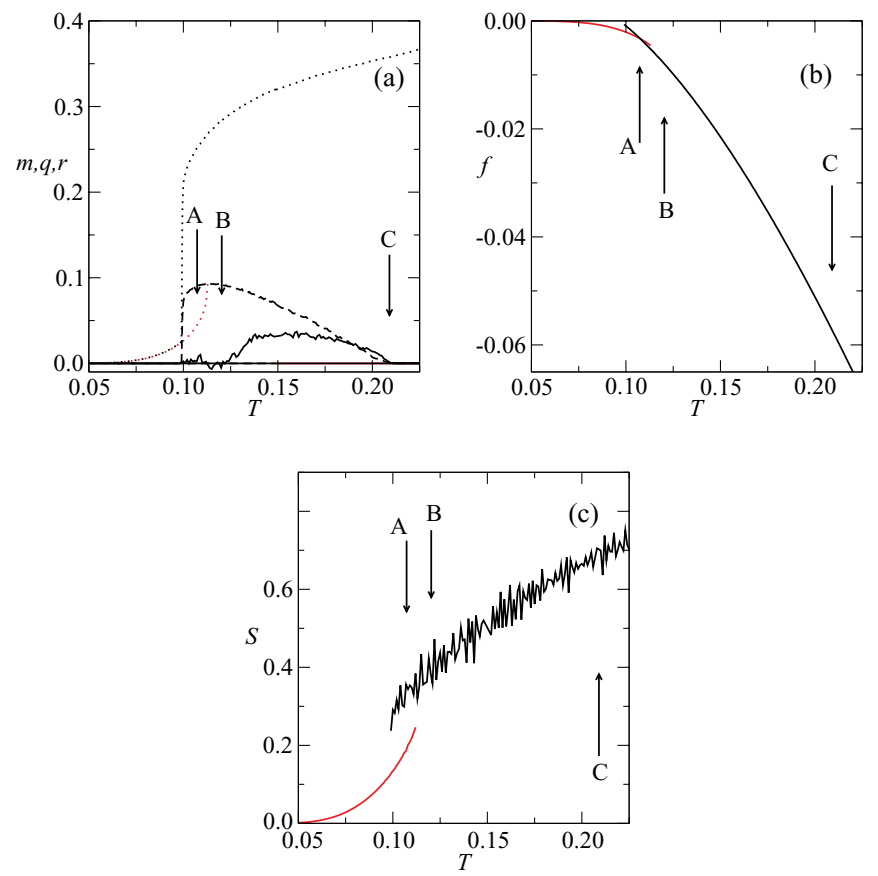

FIG. 2. (Color online) (a) Order parameters $m$ (solid line), $q$ (dashed), and $r$ (dotted) in function of the temperature for $1-p=0.24, D=0.48$, and $c=4$; (b) free-energy; (c) entropy.

to a high- $T$ ferromagnetic phase followed by an inverse freezing transition for higher disorder. The latter is the case for $1-p \geqslant 0.22$. Both inverse transitions are first-order transitions that meet the $\mathrm{F}$ to $\mathrm{SG}$ transition at a triple point. The SG phase becomes smaller when $c$ increases. Indeed, due to the normalization of the interactions with $1 / c$, the frozen-in fluctuations of the interactions that characterize the spin-glass state are reduced with increasing $c$. In contrast, the increase of the $\mathrm{F}$ phase with $c$ is favored by an increase of the alignment of the spins. In the ranges $0.22 \leqslant 1-p \leqslant 0.24$ for $c=4$ and $0.30 \leqslant 1-p \leqslant 0.32$ for $c=8$ there is a SG phase at low temperature, followed by an $\mathrm{F}$ phase at higher $T$.

In what follows, we concentrate on the regimes of small and intermediate $1-p$, where the F phase appears. Figure 2(a) shows the order parameters $m, q$, and $r$ in function of the temperature for $c=4, D=0.48$, and $1-p=0.24$. Figures 2(b) and 2(c) show the corresponding free-energy and entropy, respectively. At low $T$, the system is found in a $\mathrm{P}$ phase characterized by a low activity $r$. As $T$ increases, there is first a discontinuous transition to a SG phase, which is located by the equality of the free energies and is indicated by the arrows A between the metastable states at low $T$ (in dotted lines). It is also the place where the entropy has a discontinuity. As $T$ increases further, there is a continuous SG-F transition indicated by the arrows B. A further increase in the temperature drives continuously the system to the $\mathrm{P}$ phase of large activity $r$, indicated by $\mathrm{C}$.

Phase diagrams for $T$ versus $D$ at fixed $1-p$ and $c$ are shown in Fig. 3. Figure 3(a) corresponds to a small amount of disorder, $1-p=0.2$ for $c=4$ and $1-p=0.24$ for $c=8$. In this case there is no SG phase. There is a small range in $D$ where the phase boundary of continuous transitions has a reentrance and the system exhibits a first-order inverse melting
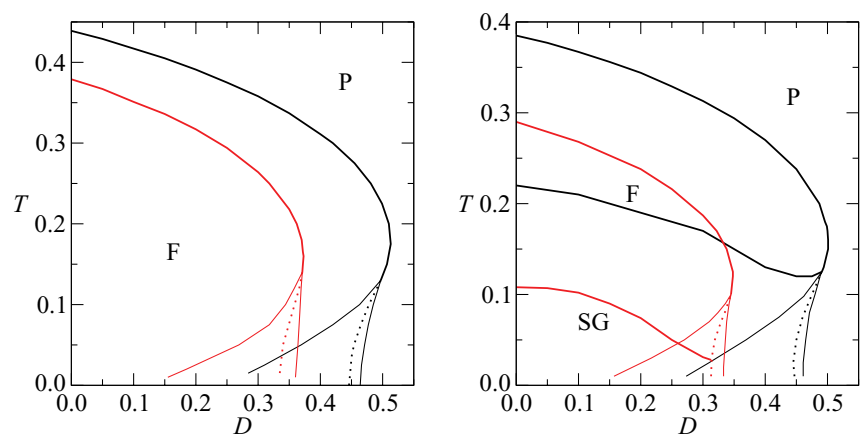

FIG. 3. (Color online) Phase diagram $T$ vs. $D$. In (a), $c=4$ and $1-p=0.2$ (black), $c=8$ and $1-p=0.24$ (red). In (b), $c=4$ and $1-p=0.24$ (black), $c=8$ and $1-p=0.31$ (red). Continuous (discontinuous) transitions are shown by heavy solid (dotted) lines and spinodals by light lines. The boundaries of each phase are discussed in the text.

transition below a tricritical point located on the reentrance. For a larger range of connectivity, $1.2 \leqslant c \leqslant 16$, the phase diagrams remain qualitatively unchanged. Thus, inverse melting and the presence of tricritical behavior seem to be robust to changes in the connectivity. If the disorder is somewhat increased, one has the situation shown in Figure 3(b). Here, $1-p=0.24$ for $c=4$ and $1-p=0.31$ for $c=8$. In both cases the continuous F-P transition has again a reentrance and a first-order transition that appears below a tricritical point. There is now also a continuous inverse transition between the $\mathrm{F}$ and SG phases, with the less ordered phase appearing at lower $T$, which seems to end at the tricritical point if $c=4$, whereas it ends at the first-order transition below the tricritical point if $c=8$. In both cases, the endpoint is a triple point where the three phases coexist.

To summarize, the phase diagrams of the three-state GS model with finite connectivity exhibiting inverse freezing in mean-field theory with RS [16] are extended in this work. Keeping in mind that finite connectivity between sites may account for effective finite-range interactions [14], we studied here the trend of the phase diagrams with the average number of connections per site. The results obtained for a range of values going from $c=4$ to a "large" $c=16$ indicate a uniform behavior of the phase boundaries with $c$ and the results obtained from mean-field theory may contain the essentials of systems with finite-range interactions.

The main features of the phase diagrams are as follows. If $D$ is small there is an $\mathrm{F}$ phase for small disorder $1-p$ and a SG phase for large disorder down to $T=0$. The continuous F-SG phase boundary has a reentrance apparently for any finite $c$ reminiscent of the fully connected Sherrington-Kirkpatrick Ising SG model [19]. For a larger $D$, the lower part of the F-SG phase boundary disappears and there is instead a low- $T$ paramagnetic phase of low-activity states giving rise to both an inverse freezing and an inverse melting transition. The presence of the low- $T$ paramagnetic phase reflects the reentrance of the phase boundary in the $T$ versus $D$ plane. From the phase diagrams, one can see that the size of the crystal field $D$ and the disorder $1-p$ play a crucial role. Similar results should follow with a Gaussian distribution for the interactions with a finite mean. 
Finally, a comment about the assumption of replica symmetry in view of the goal of the present work, which is the study of inverse transitions at not too low $T$ in the case of finite connectivity. The assumption may not be a too severe one for the transition with reentrant behavior from the low- $T$ P phase to either the F or the SG phase. Works by earlier authors on the fully connected model $[3,6]$ show that there is inverse freezing with a reentrant phase boundary even with replica-symmetry breaking (RSB) and one would expect this to be also the case for inverse melting, although the precise shape of the phase boundaries may be expected to change with RSB.

The transition from the SG to the F phase may be a different situation and, in analogy to the situation for the fully connected
Ising SG, that phase boundary could be a straight vertical line in the $(T, 1-p)$ diagram for the exact solution of the present model, with no reentrance into the F phase. However, the reader is reminded that there is only an argument and not a proof even in the case of the Ising SG [20] and apparently nothing like that yet in the case of the GS model.

The present work was supported, in part, by Conselho Nacional de Desenvolvimento Científico e Tecnológico (CNPq), Fundação de Amparo à Pesquisa do Estado do Rio Grande do Sul (FAPERGS), and Fundação de Amparo à Pesquisa do Estado do Rio de Janeiro (FAPERJ), Brazil.
[1] S. K. Ghatak and D. Sherrington, J. Phys. C 10, 3149 (1977).

[2] F. A. da Costa, C. S. O. Yokoi, and S. R. A. Salinas, J. Phys. A 27, 3365 (1994).

[3] A. Crisanti and L. Leuzzi, Phys. Rev. B 70, 014409 (2004); Phys. Rev. Lett. 95, 087201 (2005).

[4] N. Schupper and N. M. Shnerb, Phys. Rev. Lett. 93, 037202 (2004); Phys. Rev. E 72, 046107 (2005).

[5] M. Sellitto, Phys. Rev. B 73, 180202(R) (2006).

[6] M. Paoluzzi, L. Leuzzi, and A. Crisanti, Phys. Rev. Lett. 104, 120602 (2010); L. Leuzzi, M. Paoluzzi, and A. Crisanti, Phys. Rev. B 83, 014107 (2011).

[7] M. Blume, V. J. Emery, and R. B. Griffiths, Phys. Rev. A 4, 1071 (1971); W. Hoston and A. N. Berker, Phys. Rev. Lett. 67, 1027 (1991).

[8] C. V. Morais, F. M. Zimmer, and S. G. Magalhaes, Phys. Lett. A 375, 689 (2011); F. M. Zimmer, C. F. Silva, C. V. Morais, and S. G. Magalhes, J. Stat. Mech. (2011) P05026.

[9] A. Haque and E. Morris, Carbohydr. Polym. 22, 161 (1993).
[10] M. Blume, Phys. Rev. 141, 517 (1966).

[11] H. W. Capel, Physica (Amsterdam) 32, 966 (1966).

[12] C. K. Thomas and H. G. Katzgraber, Phys. Rev. E 84, 040101(R) (2011).

[13] L. Viana and A. J. Bray, J. Phys. C: Solid State Phys. 18, 3037 (1985).

[14] I. Kanter and H. Sompolinsky, Phys. Rev. Lett. 58, 164 (1987).

[15] T. Nikoletopoulos, A. C. C. Coolen, I. Perez Castillo, N. S. Skantzos, J. P. L. Hatchett, and B. Wemmenhove, J. Phys. A: Math. Gen. 37, 6455 (2004).

[16] R. Erichsen Jr. and W. K. Theumann, Phys. Rev. E 83, 061126 (2011).

[17] M. Mezard and G. Parisi, EUR. Phys. J. B 20, 217 (2001).

[18] M. Mezard, G. Parisi, and M. A. Virasoro, Spin Glass Theory and Beyond (World Scientific, Singapore, 1987).

[19] D. Sherrington and S. Kirkpatrick, Phys. Rev. Lett. 35, 1792 (1975).

[20] H. Nishimori, Statistical Physics of Spin Glasses and Information Processing (Oxford University Press, Oxford 2001). 